\title{
The Use of Modular Feed Forward Neural Networks in Anticipating the Results of Handball Championship 2015
}

\author{
Amr Hassan ${ }^{1,2}$ \\ ${ }^{1}$ Department of Sports Training, Faculty of Sports Education, Mansoura University, Mansoura, Egypt \\ ${ }^{2}$ Institute of Sport Science, University of Graz, Graz, Austria
}

Email address:

amrahh@mans.edu.eg

To cite this article:

Amr Hassan. The Use of Modular Feed Forward Neural Networks in Anticipating the Results of Handball Championship 2015. American Journal of Sports Science. Vol. 3, No. 5, 2015, pp. 93-97. doi: 10.11648/j.ajss.20150305.13

\begin{abstract}
Observation is a highly recommended approach in game analysis as it helps form a better understanding for the types of relations within the game. The aim of this study is to present a new approach for predicting competitions results which are based on game analysis by the use of Modular Forward Neural Networks (MFNN). The data of 80 games were analyzed (i.e. Fast break, Breakthrough, different type of shot...). The Data used to train Modular Feed Forward networks include 21 processing elements (PEs) as input, one element as output, 2 hidden layers, 100 epochs - termination Cross Validation, random initial weights, and weight update batch. The MFNN test contains single output case threshold 0,5 on level 1000 . Results show significant correlation between game results and neural network output $0.93,0.96$. Actual network output was 0,91 . Normalized Root Mean Square Error was 0,078. Final mean squared error was 0.9. The variables mostly affecting the results of (MFNN) were: fast breaks, and blocked shots. Using MFNN in predicting game results based on game details is considered a novel approach for evaluating the level of teams and competitors and for improving the training plans and tactics.
\end{abstract}

Keywords: Team Handball, Neural Networks, Anticipation

\section{Introduction}

During the last decade, it seems that the handball society has significantly benefited from game analysis through the regular and disciplined match analysis along with team-success determining factors of techniques, tactics and workouts. Monitoring rivals teams and evaluating them by various numeric values help re-arrange workouts. The game analysis studies in handball were classified into: Free analysis, acoustic analysis, written analysis, film analysis, video analysis, video/computer analysis and computer analysis [1]. Few literatures are interested in figuring out the relations between game details, as well as ways of investing the huge output numeric data in predicting game context. Research in game analysis has occupied a unique place in the field of sports science for a long time [2]; [3]; [4].

Neural Networks have been widely used in game analysis as they shed light on ways of using the data of game analysis effectively. Typical examples of this approach are tactical analysis and group behaviors [5]. Some literatures observed new approach of game analysis based on Custom Made Software (MASA) which is specific to determine players' positions based on tactical analysis [6]; [7]. Other literatures studied the interaction between offensive and defensive group tactics [8].

Performance and results predictions were not challenging for specialists and if this challenge took place, specialists did not resort to the use of modern methods such as advanced nonlinear modeling techniques [9] ; [10]. A substantial amount of exertion should be spent on foreseeing the results of sporting events [11]. Other researcher assumed that utilizing the neural systems helps rate and select particular players for specific rivals [12]; [13].

From this point of view the previous literatures did not pay attention to observed methods of predicting games results based on previous analytical data from game context. Therefore the aim of this study is to give new methods for anticipating game championship results by means of Modular Feed Forward Neural Networks.

The Modular Feed Forward Neural Networks (MFNN)

MFNN is considered a special class of a multilayer perceptron (MLP), which processes the input by utilizing a few parallel MLPs, and recombines the outcomes. This has a tendency to make some structures inside of the topology cultivate specialization of capacity in every sub-module. As 
opposed to the MLP, particular systems don't have a full interconnectivity between their layers. Thus, a fewer number of weights are needed for the same size system (i.e. the same number of PEs). This has a tendency to accelerate preparing times and to diminish the quantity of obliged preparing models. There are numerous approaches to portion an MLP into the module. It is hazy how to plan the measured topology in light of the information. It cannot be certain that every module is practicing its preparation for a one of a kind part of the data [14];[15].

\section{Material \& Methods}

Out of 160 half games from the handball world cup championship 2015, 149 half games were analyzed, the only half - games finished with a win or lose were considered, no equal games are included in this study. Eighteen quantity / quality game variables were determined as shown in table (1).

Table 1. Quantity / Quality game variables and desired results of half games for each team.

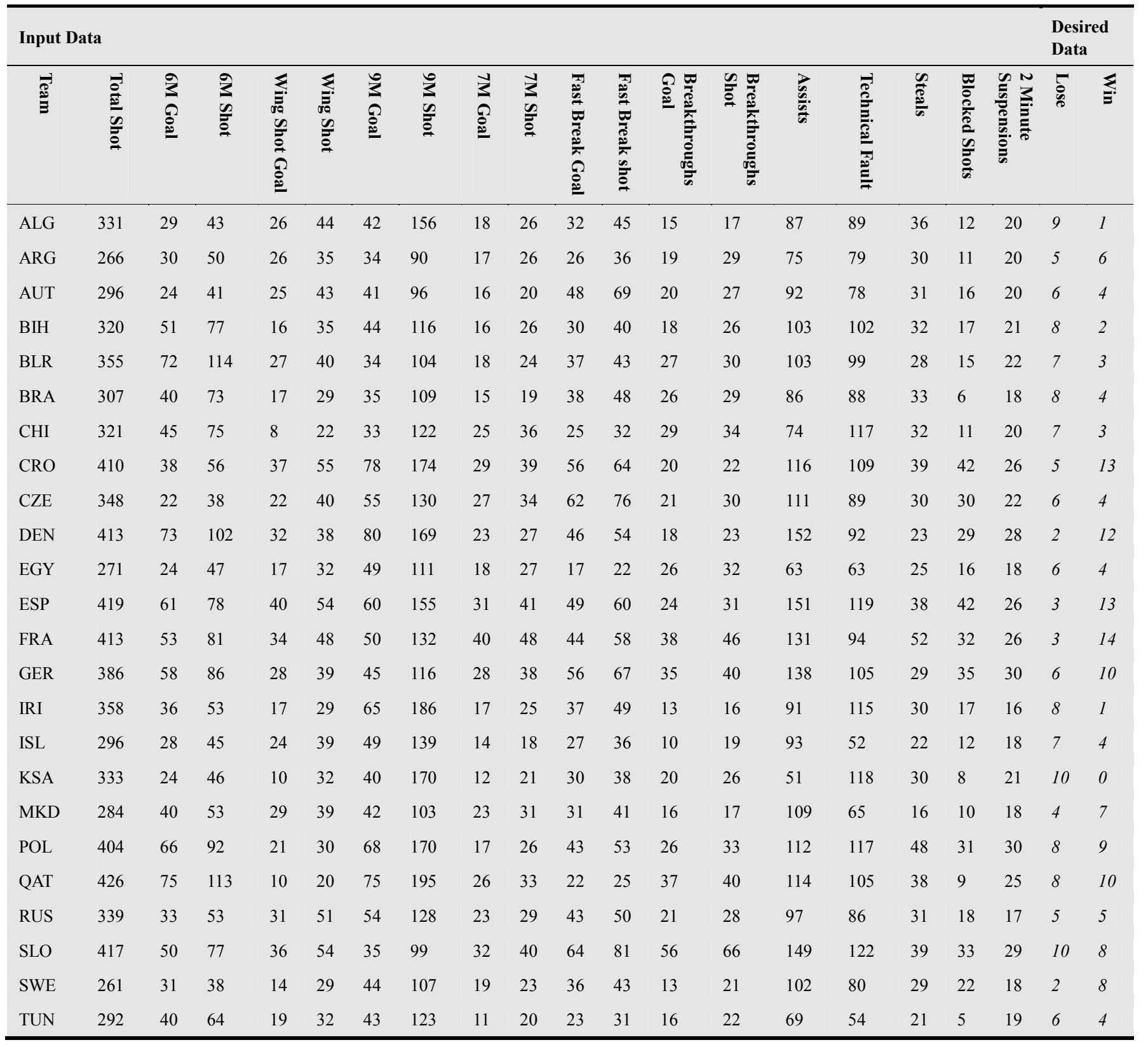

Network Design, Training and Test

The network design and training procedures contain 21 PEs as input, one as output, sixteen exemplars, two hidden layers, 100 Epochs Termination Cross Validation, random initial weights and weight update batch. The MFNN test contains single output case threshold 0.5 on level 1000 Epochs (as shown in fig 1). 


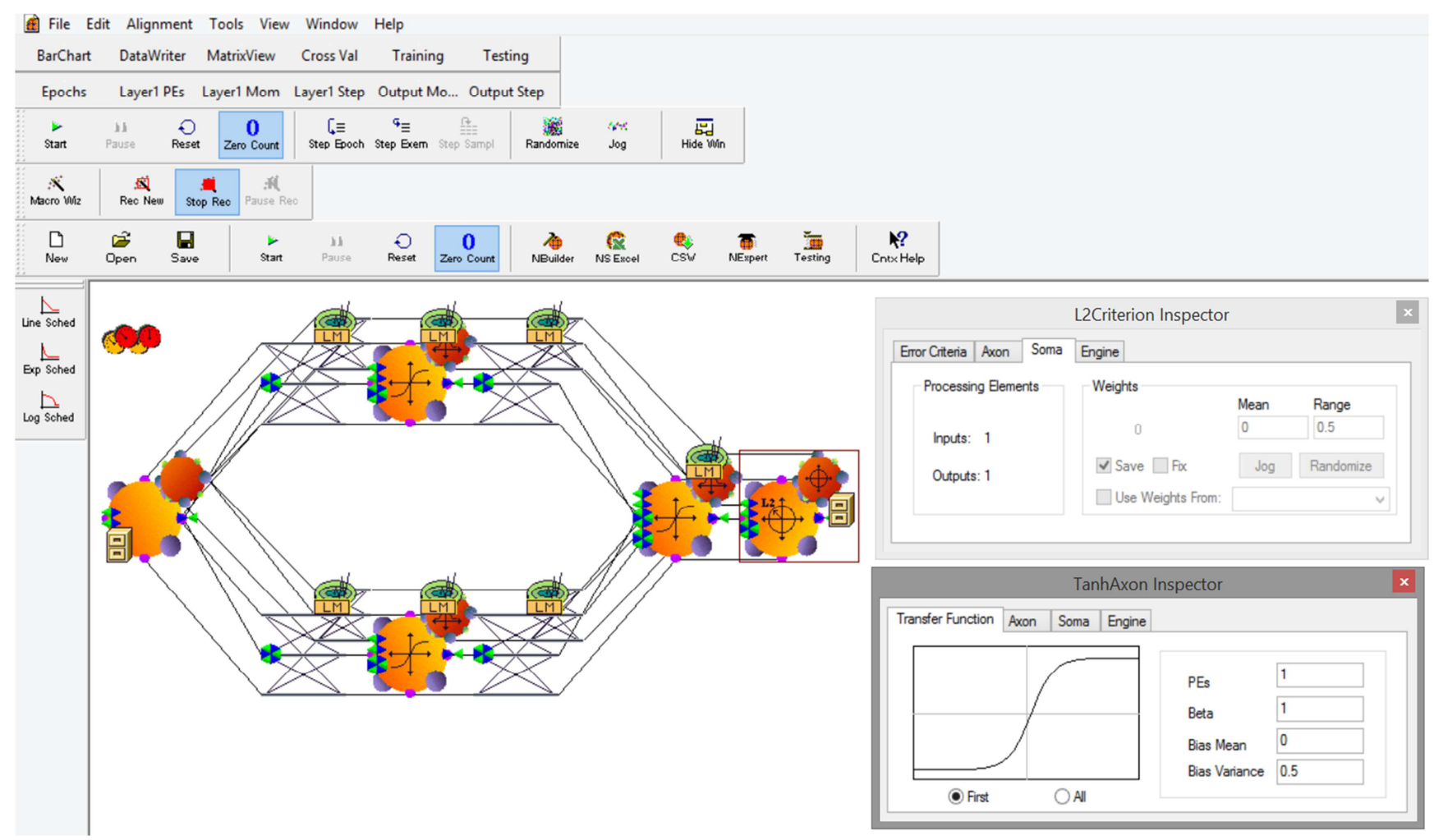

Figure 1. Graphical user interface of the (MFNN).

The outputs of championship analysis (eighteen quantity / quality variables) were used to feed the neural network as input data, also game results (win / lose) were marked as desired data. Up to $70 \%$ of all data were marked as training, while $15 \%$ were marked as cross validation. Cross validation processes the lapse in a test set while the system is being prepared with the preparation set. It is realized that the mean squared normalized error (MSE) will continue to diminish in the preparation set, however, it may begin to increase in the test set. This happens when the system begins "remembering" the preparation designs. The Termination page of the enactment control reviewer can be utilized to screen the cross approval set mistake and consequently it stops the system when it is not progressing. $15 \%$ of all data were marked as testing data. The sufficient correlation between desired and output results from the neural network was calculated. The network train report also contains minimum training MSE at last epoch (table. 2).

Table 2. Mean squared normalized error for training and cross validation.

\begin{tabular}{lll}
\hline Best Networks & Training & Cross Validation \\
\hline Epoch \# & 8 & 1 \\
Minimum MSE & $5.74129 \mathrm{E}-29$ & 0.022651597 \\
Final MSE & $5.74129 \mathrm{E}-29$ & 0.053782498 \\
\hline
\end{tabular}

\section{Results}

There is no significant different between the mean of actual championship results and the MFNN results. $\mathrm{P}$ value was 0.005 in case of win or lose as show in table 3 .
Table 3. The mean and standard deviation of actual and (MFNN) output data, $P$ values (2-tailed test) also shown.

\begin{tabular}{llllll}
\hline Half games & \multicolumn{2}{l}{ Actual data } & \multicolumn{2}{l}{ Network output } & P \\
\hline \multirow{2}{*}{ Win } & Mean & SD & Mean & SD & \\
Lose & 6,2 & 1.1 & 5.58 & 0.97 & 0.005 \\
\hline
\end{tabular}

Test report on network performance shows values of Root Mean Square Error (RMSE), it was between 2.5 in case of win and 2.3 in case of lose. Normalized Root Mean Square Error (NRMSE) and Mean Absolute Error (MAE) are also shown. Correlation between the actual results and the MFNN results was very high. In case of win, it was 0.930 and in case of lose, it was 0.960 as shown in table 4 .

Table 4. The test report error of the neural network shows the sufficient correlation between desired competition results (win / lose in each half game) and (MFNN) output data.

\begin{tabular}{lll}
\hline Performance & Win & Lose \\
\hline RMSE & 2.517 & 2.316 \\
NRMSE & 0.209 & 0.217 \\
MAE & 2.272 & 2.649 \\
Min ABS Error & 0.180 & 0.183 \\
Max ABS Error & 3.382 & 3.303 \\
R & 0.930 & 0.960 \\
\hline
\end{tabular}

The accuracy of actual competition results and the MFNN output are shown in fig 2 and the game data affecting the MFNN accuracy are shown in fig 3. 


\section{Desired Output and Actual Network Output}

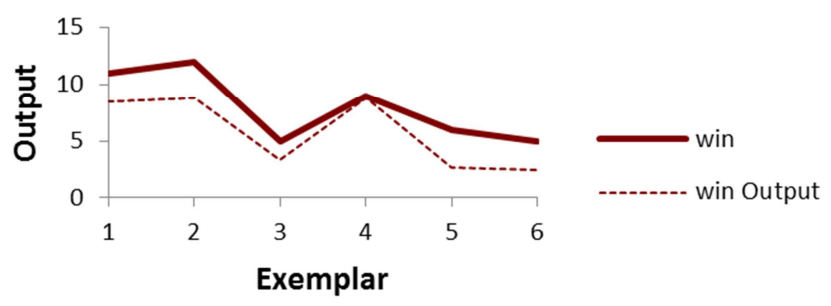

Figure 2. Championship game results and actual (MFNN) output. (I.e. Win for each half game)

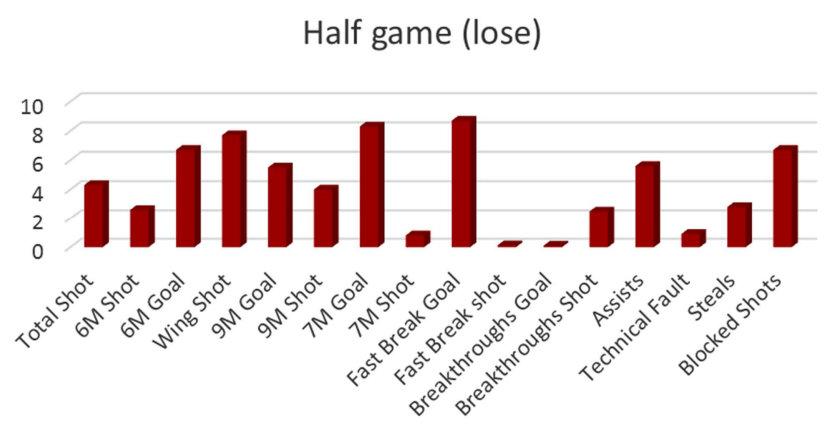

Figure 3. The Data of Half Game (Lose) Affecting (MFNN) output.

\section{Discussion}

The previous presentation of the results shows the reliability of the MFNN methodology in predicting the results of games in the competition. Previous studies relied on the prediction of athletic performance only without the possibility of pre-research to identify the results of matches, therefore, they used a hybrid prediction system based on genetic algorithm and artificial neural network (GANN) [12], or the neural networks to select players. Both are based on tests in advance [13].

This study tried to anticipate the results of the handball world cup championship 2015 by using modular feed forward neural networks and relied on neural network feed variables resulting from the analysis of the tournament and number 18 variables from game events, The results show that there are no significant differences between the expected output of the MFNN and the actual data of the competitions. Results also showed the presence of a high correlation between the output results by using MFNN and actual results for each half game in all cases of winning and losing as 93\% and 96\%, respectively.

Also, the mean square error of the neural network was at its lowest value 1.8, which increased the confidence in the results presented. In the same context, the sensitivity of MFNN input data to game (win/lose) will help trainers and experts to understand the nature of the biggest factors influencing the outcome of matches and thus they work to take advantage of playing through the development of training programs and plans. Another point of view adopts the possibility of the use of MFNN to evaluate team performance in a phased manner during the competitions. It will identify the level at which the team is heading and its competitors. This will help us make quick decisions that will change the course of the results and the team.

The prospective values of this study is to show that the approach of artificial Feed Forward Neural network dealing with the methods of optimal use of the data in the field of sports based on performance analysis is a reliable approach in predicting game results. The performance output data are necessary as a base to predict other values in cooperation with MFNN. Using this proposed methodology of MFNN helps predict large values which in turn will lead us to improve the player's performance, (I. e.) Type of tactics.

\section{Conclusion and Future Work}

The Modular Forward Neural Network was used for game execution forecast. The test results can help the trainers to nearly foresee the performance of the team and the competitors. Any future research work ought to look at some genuine information from distinctive games and diverse levels. Moreover, the researchers ought to enhance the calculation to make the expectation more exact. At long last, it ought to look at different methodologies for expectations.

\section{References}

[1] Y. Taskiran. (2007). "2007 EHF Youth Coaches' Course during the 2007 W 19 European Championship," Coach. E H F Youth Championship, Eur.

[2] A. Hohmann and M. Lames. (2005). "Trainings-und Wettspielanalyse," M. K. \& K. R. In A. Hohmann and (Hrsg.), Eds. Handbuch Sportspiel. - Schorndorf: Hofmann, pp. 376-394.

[3] D. Memmert and K. Roth. (2003). "Individualtaktische Leistungsdiagnostik im Sportspiel,” Spectrum, vol. 15, no. July, pp. $44-70$.

[4] W. Schöllhorn and P. Jürgen. (2002). "Prozessanalysen in der Bewegungs- und Sportspielforschung - Sportinformatik," Spectr. der Sport., vol. 14, no. 1, pp. 30-52.

[5] M. Pfeiffer and J. Perl. (2006). "Analysis of tactical Structures in team handball by means of artificial neural networks," Int. J. Comput. Sci. Sport, vol. 5, no. 1, pp. 4-14.

[6] P. Rudelsdorfer, N. Schrapf, H. Possegger, T. Mauthner, H. Bischof, and M. Tilp. (2014). "A novel method for the analysis of sequential actions in team handball," Int. J. Comp. Sci. Sport, vol. 13 , no. 1 , pp. $69-84$.

[7] N, Scharpf., M, Tilp. (2013). “Action sequence analysis in team handball," J. Hum. Sport Exerc. North Am., vol. 8, no. 3Proc, pp. 615-621.

[8] S, Alsaied., N, Scharpf., A, Hassan., M, Tilp. (2015). “Analysis Of Interaction Between Offense And Defence Tactics In Team Handball By Means Of Artificial Neural Networks," in 20th Annual ECSS-Congress, Malmö.

[9] M. Janta, C. Ebert, and V. Senner. (2012). "Functionality and performance of customized sole inlays for various sports applications," Procedia Eng., vol. 34, pp. 290-294. 
[10] Y. Torun and G. Tas. (2012). "Remote Sensing Image Classification By Non-Parallel Svms," Adv. Inf. Technol. andManagement, vol. 1, no. 3, pp. 1-3.

[11] H. O. Stekler, D. Sendor, and R. Verlander. (2010). "Issues in sports forecasting," Int. J. Forecast., vol. 26, no. 3, pp. 606-621.

[12] X. Biao. (2012). "Prediction of Sports Performance based on Genetic Algorithm and Artificial Neural Network," Int. J. Digit. Content Technol. its Appl., vol. 6, no. 22, pp. 141-149.
[13] S. R. Iyer and R. Sharda. (2009). "Prediction of athletes performance using neural networks: An application in cricket team selection," Expert Syst. Appl., vol. 36, no. 3, pp. $5510-5522$.

[14] V. G. Ivancevic and T. T. Ivancevic. (2010). Quantum Neural Computation. Springer Science \& Business Media,.

[15] A. O. Ali, I. A. Saleh, and T. R. Badawy. (2010). "Intelligent Adaptive Intrusion Detection Systems Using Neural Networks ( Comparitive study )," Int. J. Video\& Image Process. Netw. Secur. IJVIPNS-IJENS, vol. 10, no. 01, pp. 1-8. 